\title{
Real-time Patient Healthcare Telemonitoring using Zigbee
}

\author{
Authors \\ Dipak Raut ${ }^{1}$, Anjali Jagtap ${ }^{2}$, Smita Kadam $^{3}$, Ashok Shinde 4 , Parag Hirulkar \\ ${ }^{1}$ International Institute of Information Technology, Pune, India, Email: dipakr@isquareit.edu.in \\ ${ }^{2}$ International Institute of Information Technology, Pune, India, Email: anjalijagtap2306@gmail.com \\ ${ }^{3}$ International Institute of Information Technology, Pune, India, Email: smitak@isquareit.edu.in \\ ${ }^{4}$ International Institute of Information Technology, Pune, India, Email: ashoks@isquareit.edu.in \\ ${ }^{5}$ International Institute of Information Technology, Pune, India, Email: paragh@isquareit.edu.in
}

\begin{abstract}
In recent years, the focus of health policy has been shifting from reactive and acute healthcare in hospital towards providing more proactive and preventive healthcare. Because emergency admission to hospital and continuous check up at hospital are extremely costly, and do challenge the available medical resources, the mode of healthcare is being innovated to reduce and avoid this circumstances. In fact the reason for staying in hospital in most cases is not because a patient actually needs continuous observation for reliably detecting the progressive abnormalities, timely monitoring therapy effect or avoiding occurrence of adverse events in recovery process of post surgery. Meanwhile, early detection of health anomalies can greatly reduce the emergency admissions to large acute centers. Therefore, medical and health societies are seeking the technologies to be able to advance healthcare by early detection of health anomalies and by early discharge of patients from hospital with continuous observation. This will not only reduce the risk of crossinfection, but also significantly decrease the cost of hospitals
\end{abstract}

Keywords-PPG, ZigBee, Telemonitoring, Patient Monitoring, HMS.

\section{Introduction}

Since the photoplethysmogram (PPG) technology is mature, simple, and low cost, the PPG has been widely applied in many kinds of physiological information monitor and the guardianship instruments. This system takes the PPG as the basic method in the measurement of heart rate and the ZigBee wireless communication protocol as the network platform. This physiological information monitor system can realize the real-time physiological information collection, transmission, save and report in the certain scope. It provides a kind of more effective solution for the community guardianship. In the system proposed here, the patient health is continuously monitored by the sensors kept in contact with his body and the acquired data is transmitted to a centralized controller using Wireless Sensor Networks. A ZigBee node is connected to every patient monitor system which consumes very low power and is extremely small in size. The paper is organized in a neat way with explanation on temperature sensor, heart beat sensor, power sources and the detailed understanding of microcontrollers. Next, ZigBee has been discussed with the Architectural model of the complete system. Then, the circuit design of heartbeat sensor and the software implementation 
are presented followed by conclusions and the references. The typical objectives of the system are as mentioned below.

\section{Goals \& Objective}

There are certain issues involved in the design of this system. The system should be scalable, so that new device can easily be integrated into it. It should provide a user-friendly interface on the host side, so that the devices can be setup, monitored and controlled. The interface should also provide some diagnostic services so those problems with the system, if any, can be tracked down. The overall system should be fast enough to realize the true power of wireless technology. It should also be cost effective in order to justify its application.

- To design a real-time health monitoring system based on wireless technology.

- To continuously monitor patient's body temperature \& heartbeat rate.

- To study the principle of photoplethysmography and the types of heartbeat sensors

- To design and interface the circuit of heartbeat sensor

- To study the utilization and application of ZigBee technology.

- To manage and organize a patient's information and diagnosis in a hospital

- To study and design the hardware-software phases of the system.

\section{Analysis of Existing Literature}

Wireless sensor network has proved to be best in remote monitoring of patient's health. Furthermore, integrating diverse medical sensors together can combine medical information from multiple sources, thus improving diagnostic accuracy of a disease, simplifying diagnostic procedure, and discovering potential knowledge through data fusion of all gathered information. Actually, recent technology advances in wireless communication, micro-power medical sensors, and network technologies make it possible to design the new-generation health-care system, which brings together the abundance of existing specialized medical technology with pervasive, wireless networks, and low cost, miniature, lightweight, intelligent physiological sensors ${ }^{[1]}$

In this section, literature survey of existing system is done to make the analysis and design new system. Ho Ting Cheng and Weihua Zhuang ${ }^{[2]}$ introduced a Bluetooth-enabled in-home Patient Monitoring System for early detection of Alzheimer's disease. They have used short range. Bluetooth communications for in-home patient location tracking, and then the location information was recorded in a local database. With the knowledge of the movement pattern of a patient, a medical practitioner is more likely to be able to determine whether a target patient is developing Alzheimer's disease.

N. S. R. K. Prasad and A. Rajesh ${ }^{[3]}$ created standards-based secure access to patient's personal data and medical records by using RFID tags and Web Service with the help of hardware kit. This system uses Web service interfaces to support standard Electronic Health Records for patient record interoperability. Customers can view and update their personal medical information via the web site, which seamlessly sync with one another. Because the system is built on Web services, it is easy to update, adapt and grow.

Naazneen M. G. et al. ${ }^{[4]}$ described the design of an ECG monitoring system which monitors ECG and heart rate measurement system which measures the heart rate of the subject by $\mathrm{Ag} \mathrm{/} \mathrm{Cl}$ sticking electrode on the arms and then displaying the ECG on cathode ray oscilloscope (CRO) and heart rate on a text based LCD. The device has the advantage that it is microcontroller based and thus can be programmed to display various quantities, such as the normal, maximum and minimum rates over a period of time and so on. Another advantage of such a design is that it can be expanded and can easily be connected to a recording device or a PC to collect and analyze the data for over a period of time.

K. Navya and Dr. M. B. R. Murthy ${ }^{[5]}$ presented a Wireless Sensor Network (WSN) for monitoring patient's physiological conditions continuously 
using Zigbee. The same is transmitted to the remote wireless monitor to observe patient's physiological signal. The remote wireless monitor is constructed of Zigbee and Personal Computer (PC).

Ying Zhang and Hannan Xiao ${ }^{[1]}$ described the overall system architecture of their developed Bluetooth sensor network for patient monitoring and the corresponding heart activity sensors. They also presented the approach to developing the intelligent physiological sensor nodes involving integration of Bluetooth radio technology, hardware and software organization, and the solutions for onboard signal processing. The collected data is transmitted to a medical center. National Semiconductor's CP3SP33 connectivity processor is adopted to design the sensor node and the coordinator node.

Electrogastrography (EGG) is a non-invasive method for recording the electrical activity of the stomach. The paper presented by S. Haddab and M. Laghrouche ${ }^{[6]}$ consisted of a system designed for monitoring the EGG physiological variables of a patient outside the hospital environment. The signal acquisition was achieved by means of an ambulatory system carried by the patient and connected to him through skin electrodes. Using Bluetooth technology, data is transferred for processing and diagnostic unit in the hospital. EGG is usually contaminated by artefacts and other signals, which are sometimes difficult to remove. Hence, a neural network method was used for motion artefacts removal and biological signal separation.

Vipin Gupta et al. ${ }^{[7]}$ put forward a system for the measurement of Heart Rate, Temperature and Blood Oxygen level using Low Cost Microcontroller. The temperature sensor used was DS18B20 digital sensor. Heart rate and arterial blood oxygen was combined using photo-plethysmography (PPG) technology. System hardware included three basic parts: one is temperature Sensor, other is optical sensors and its signal conditioning circuit to detect Spo2 and heart rate together and the third one is microcontroller where all computational work was done.
Subhani Sk. M. et al. ${ }^{[8]}$ implemented GSM based Heart Rate and Temperature Monitoring System in which heartbeat and temperature of patient were measured by using sensors as analog data. It was then transmitted wirelessly using paging messages through GSM modem. AT89S52 microcontroller device was used for temporary storage of the data used for transmission. In order to communicate with the GSM modem, they have used a special set of commands called SMS AT Commands.

\section{System Block Diagram}

The block diagram consists of the system transmitter module and receiver module. It shows the principal parts with their functional flow and relationships between them. It can be divided into three phases:

\section{Monitoring Phase}

When a patient is admitted to the hospital, the doctor will set the patient's bed number and his admission date into the transmitter unit with the help of the touchpad. Then the sensors will be kept in contact with the patient's body. The temperature sensor will measure the temperature in degree centigrade by converting the temperature data into the linear output analog voltage while the heartbeat sensor will measure the heartbeat rate in beats per minute using photo-plethysmography principle. However, the signal obtained is weak and noisy. Hence, initially it is filtered and amplified and then sent to the microcontroller. These two physiological parameters of the patient's body will be continuously monitored.

\section{Processing Phase}

This phase consists of only the microcontroller. The microcontroller is the heart of the system. It performs the following operations in transmitter module:

- Accepting the physiological data from sensors

- Converting analog voltage from temperature sensor to digital form using inbuilt $\mathrm{A} / \mathrm{D}$ converter.

- Counting the heartbeats pulses. 
- Converting processing data into ASCII format.

- Displaying the parameters on transmitter LCD module.

- Transmitting the data serially through ZigBee module.

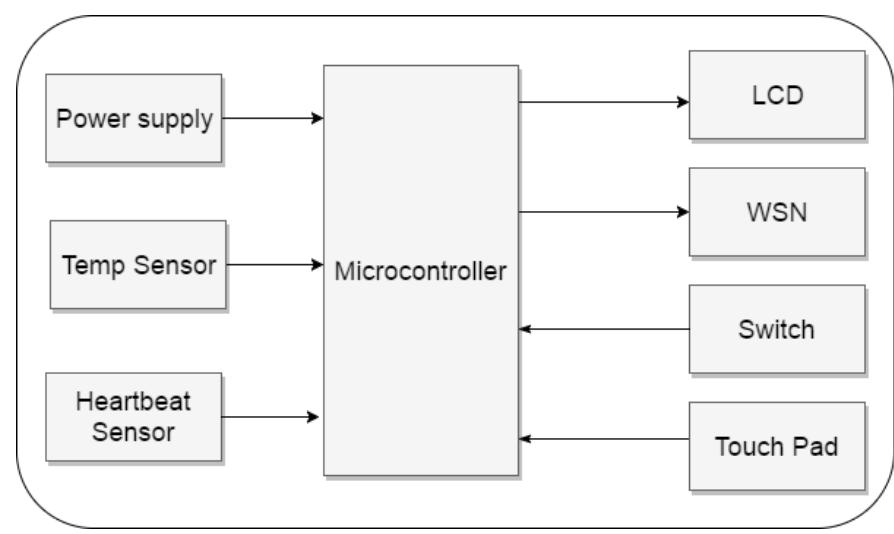

Fig.1. Transmitter Module

\section{Display Phase}

This receiver module consists of ZigBee module for reception of the data which is further given to the microcontroller. The microcontroller stores the data into an access database that can be the controller's own memory or an external memory interfaced to the microcontroller. The values can then be the controller's own memory or an external memory interfaced to the microcontroller. The values can then be displayed on the LCD module interfaced to the controller. Once the user has connected to the receiver unit, data is automatically updated on the display.

Patient's bed number, his heart beats per minute (BPM), his body temperature and his admission date in the hospital are displayed on LCD. The design is modular which makes it easy and straight forward to add any extra sensors for measuring and monitoring the other parameters.

If it is inferred that the person is medically distressed, an alarm may be generated by pressing a switch at the transmitter module along with an emergency message displayed on LCD. The LCD displays a report on the current status of the individual.
Hardware \& Software

Hardware Requirements

System Specifications

- Microcontroller - Atmega 16

- Temperature sensor-LM35

- Heartbeat sensor - Reflection type

- Maximum measurable heartbeat: 150 bpm

- ZigBee module range: $30-40 \mathrm{~m}$

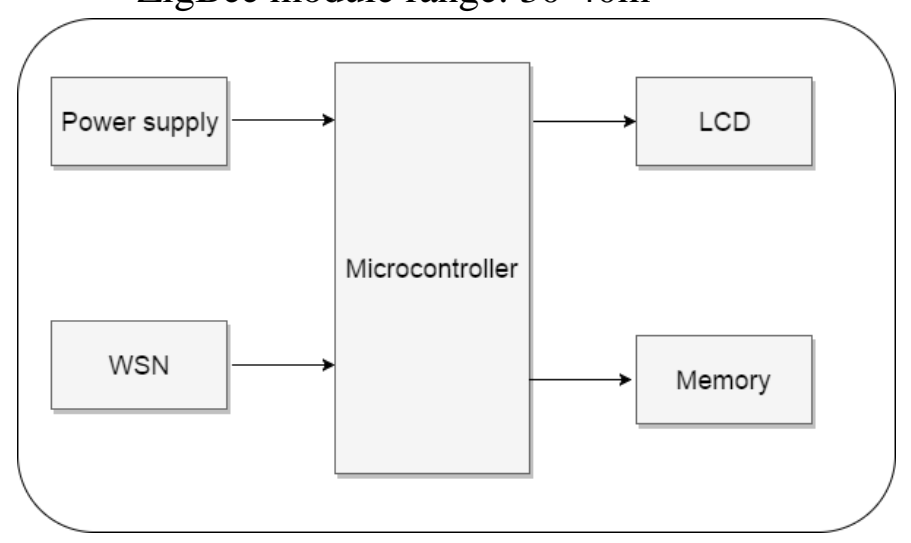

Fig.2. Receiver Module

\section{Software Requirement}

Compilers produce hex files that we download into the flash of the microcontroller. The size of the hex file produced by the compiler is one of the main concerns of microcontroller programmers because microcontrollers have limited on-chip flash. The question arises that how the choice of programming language affects the compiled program size.

For simulation purpose Proteus 7 was used. PCB is designed using Eagle and coding is done in Atmel studio. Sinaprog is hex downloader application with AVR Dude and Fuse bit calculator. It is used to download code or program and to set fuse bits of an AVR microcontroller.

\section{Result \& Analysis}

The transmitter module was implemented in Proteus software. The output voltage of temperature sensor was applied to the channel 0 of inbuilt ADC. The control pins were applied to port B while the data pins were applied to port $\mathrm{C}$. The pulses were applied through a pulse generator to the channel 1 of inbuilt ADC. The reference voltage of $2.56 \mathrm{~V}$ was applied through internal supply of microcontroller. The data was simultaneously transmitted through the virtual terminal. The following figure shows the simulation 


\section{JMSCR Vol||05||Issue||06||Page 23792-23797||June}

results. The heartbeat rate and temperature of patient's body is taken from sensors. After pressing the key data is sent through Zigbee module and the message is displayed on the screen.

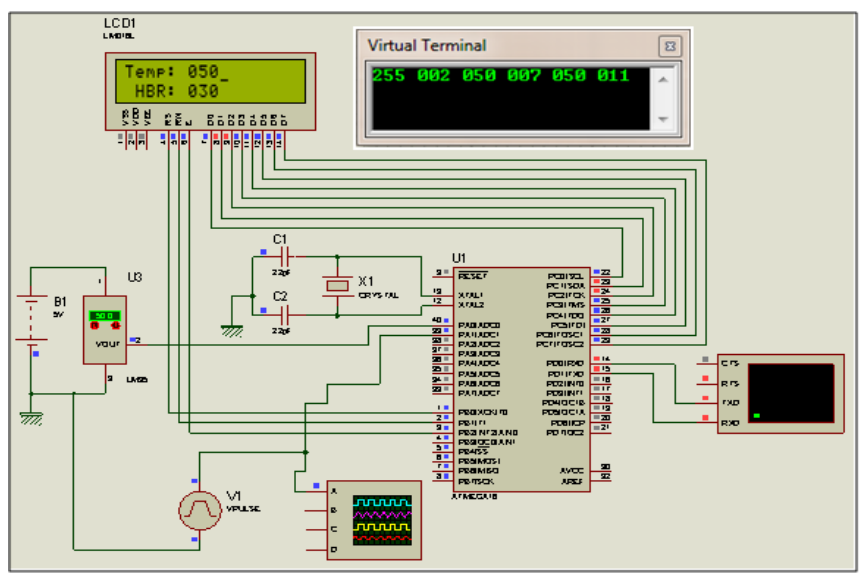

Fig.3.Simulation result
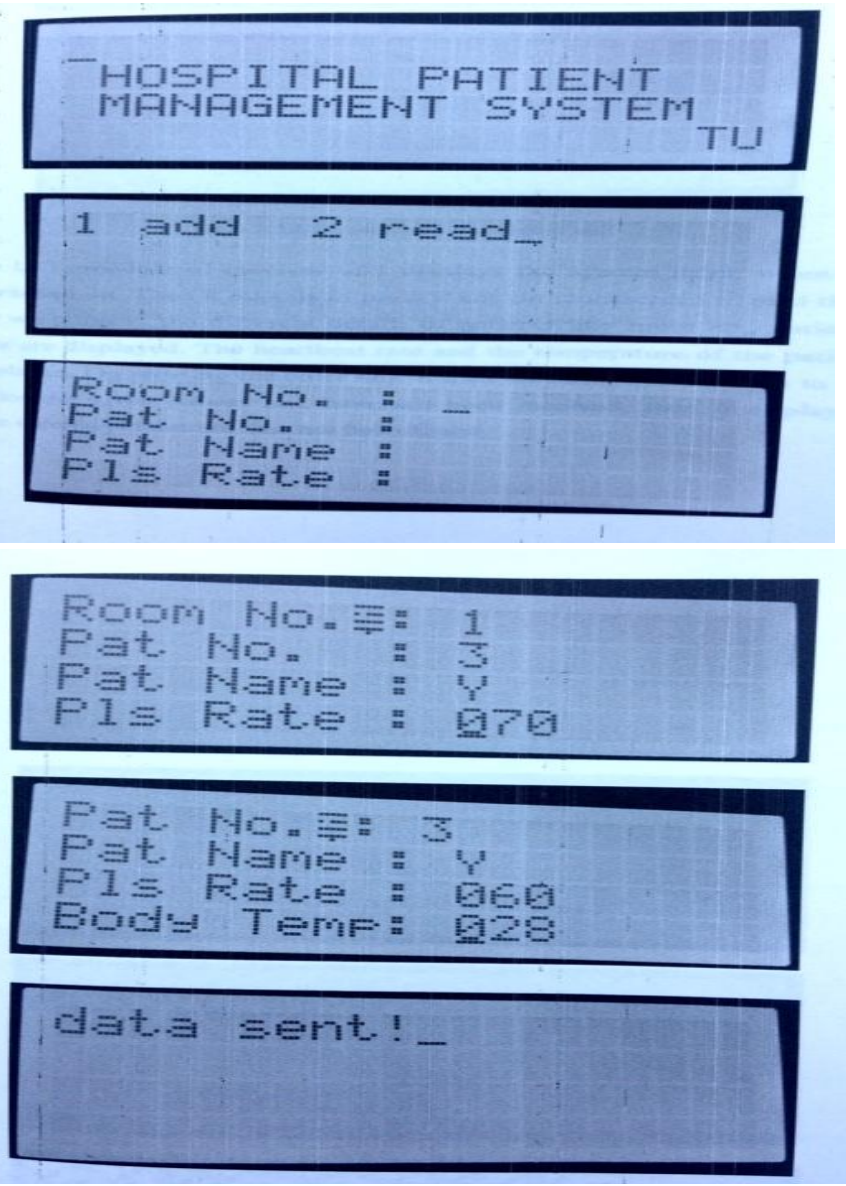

Fig.4. LCD output on transmitter unit

Thus the system enables reliable remote patient monitoring with improved quality and reduced risk. It allows continuous real time data monitoring without any computer or manpower.

\section{Conclusion}

Due to the systematic and sophisticated implementation of the hardware as well as software required to design the proposed system, we can conclude that the system exhibits the following characteristics and features as described below:

- Simplicity: The system architecture is a simple one with no complex system or communication architecture. Even the coding for the system is easy if done in embedded $\mathrm{C}$.

- Cost effective: The system consists of some low cost sensors. The communication from sensors is also cheap due to the use of low cost ZigBee technology.

- Security: Security is a major issue in health monitoring system. Suppose a patient is suffering from a critical condition like increase in the body temperature. The emergency message along with the buzzer in the doctor's cabin will help to take immediate actions to help the patient to recover.

- Prevention of paper wastage: In conventional hospital management methods, lots of paper is used to maintain the patient's health related records. In the proposed system, the paper wastage will be prevented since an external memory has been interfaced to receiver controller to update the database of the patients.

- Wireless data transmission: The patient's physiological condition is transmitted using wireless ZigBee technology which consumes less power.

HQSFITFL FATIENT MPFHAEREMT

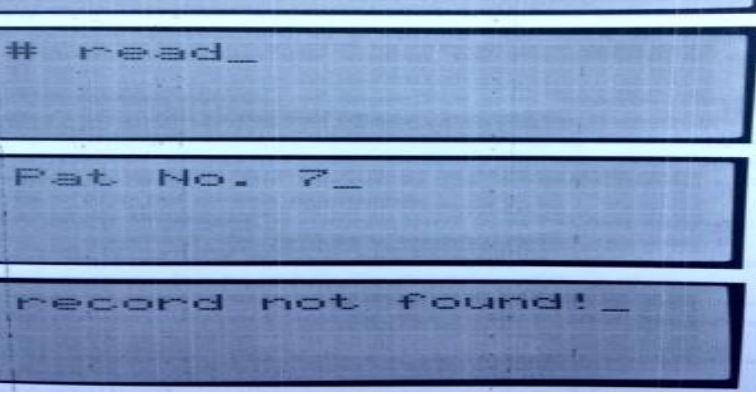

Fig.5.LCD output on receiver unit 
- Coverage range: The ZigBee module has higher range of about $200 \mathrm{~m}$ as compared to the Bluetooth which has the range of only 10 $\mathrm{m}$

\section{References}

1. Ying Zhang and Hannan Xiao, "BluetoothBased Sensor Networks for Remotely Monitoring the Physiological Signals of a Patient," IEEE Trans. Information Technology in Biomedicine, Vol. 13, No. 6, Nov. 2009.

2. Ho Ting Cheng and Weihua Zhuang, "Bluetooth-enabled In-home Patient Monitoring System: Early Detection of Alzheimer's Disease," IEEE Wireless Communications, Feb. 2010.

3. N. S. R. K. Prasad and A. Rajesh, "RFIDBased Hospital Real Time Patient Management System," International Journal of Computer Trends and Technology, vol. 3, issue 3, 2012.

4. Naazneen M. G. et al., "Design and Implementation of ECG Monitoring and Heart Rate Measurement System," International Journal of Engineering Science and Innovative Technology, vol. 2, issue 3, May 2013.

5. K. Navya and Dr. M. B. R. Murthy, "A Zigbee based Patient Health Monitoring System," International Journal of Engineering Research and Applications, vol. 3, issue 5, pp. 483-486, Sep-Oct 2013.

6. S. Haddab and M. Laghrouche, "Microcontroller-based System for Electrogastrography Monitoring through Wireless Transmission," Measurement Science Review, Vol. 9, 2009.

7. Vipin Gupta, "Design and Development of Anti-Spoofing based measurement of Heart Rate, Temperature and Blood Oxygen level using Low Cost Microcontroller," International Journal of Electronics and Computer Science Engineering.
8. Subhani Sk. M. et al., "Implementation of GSM Based Heart Rate and Temperature Monitoring System," Research Journal of Engineering Sciences, Vol. 2(4), April 2013.

9. M. A. Mazidi and S. Naimi, The AVR Microcontroller and Embedded Systems Using Assembly and C. India: Pearson, 2014. 